\title{
Перспективы использования гексаборидов редких земель в термоэлектрических однофотонных детекторах
}

\author{
(C) А.С. Кузанян ${ }^{1}$, А.А. Кузанян ${ }^{1}$, В.Р. Никогосян ${ }^{1}$, В.Н. Гурин ${ }^{2}$, М.П. Волков ${ }^{2}$ \\ ${ }^{1}$ Институт фризических исследований Национальной академии наук Армении, \\ 0203 Аштарак, Армения \\ ${ }^{2}$ Физико-технический институт им. А.Ф. Иоффе Российской академии наук, \\ 194021 Санкт-Петербург, Россия \\ E-mail: akuzanyan@yahoo.com
}

(Получена 27 декабря 2016 г. Принята к печати 12 января 2017 г.)

Анализируются результаты компьютерного моделирования процессов распространения тепла, протекающих после поглощения одиночных фотонов с энергией 1 эВ-1кэВ в трехслойном сенсоре термоэлектрического детектора. Рассмотрены различные геометрии сенсора с вольфрамовым поглотителем, термоэлектрическим слоем из гексаборида церия и вольфрамовым теплоотводом. Показано, что посредством изменения геометрических размеров слоев сенсора можно получать датчики для регистрации фотонов конкретного спектрального диапазона с необходимыми энергетическим разрешением и скоростью счета. Сделан вывод, что трехслойный сенсор имеет ряд преимуществ по сравнению с однослойным сенсором и обладает характеристиками, позволяющими рассматривать термоэлектрический детектор как реальную альтернативу сверхпроводящим однофотонным детекторам.

DOI: $10.21883 /$ FTP.2017.07.44639.25

\section{1. Введение}

Однофотонные детекторы, позволяющие определять энергию фотона и обеспечивать высокую скорость счета, востребованы в квантовой электронике, космической астрофизике, физике высоких энергий, квантовой информатике, телекоммуникационных системах, квантовой метрологии, компьютерной томографии и других областях [1]. Среди разработок последних 15 лет наиболее перспективными считаются сверхпроводящие нанопроволочные однофотонные детекторы (SNSPD) [2], которые превосходят по своим характеристикам все ранее известные детекторы. Похожими характеристиками обладает термоэлектрический однофотонный детектор (TSPD), физическая концепция которого была предложена в 2000 г. [3] и явилась единственно новым подходом в методах детектирования одиночных фотонов за довольно продолжительное время. Дальнейшие исследования показали, что TSPD могут составить конкуренцию SNSPD, так как превосходят их по некоторым характеристикам [4]. Цикл исследований процессов распространения тепла в однослойном сенсоре термоэлектрического детектора с помощью компьютерного моделирования выявил ряд особенностей TSPD, в частности зависимость отклика от области термализации фотона в поглотителе (абсорбере). Недавно была предложена идея трехслойного сенсора TSPD, которая обеспечивает независимость отклика от области термализации фотона [5]. В данной работе приводятся результаты компьютерного моделирования процессов распространения тепла в трехслойном сенсоре термоэлектрического детектора, протекающих после поглощения фотонов с энергией от 1 эВ до 1 кэВ.

\section{2. Сенсор термоэлектрического детектора и методика эксперимента}

Однослойный сенсор TSPD содержит два поглотителя из тяжелого металла, которые нанесены на диэлектрическую подложку и соединены друг с другом термоэлектрическим мостиком (рис. $1, a)$. Когда в один из поглотителей попадает фотон, его температура повышается по сравнению с другим поглотителем, на термоэлектрическом мостике возникает разность потенциалов, измеряя которую, можно не только зарегистрировать факт поглощения фотона, но и определить его энергию. Работа

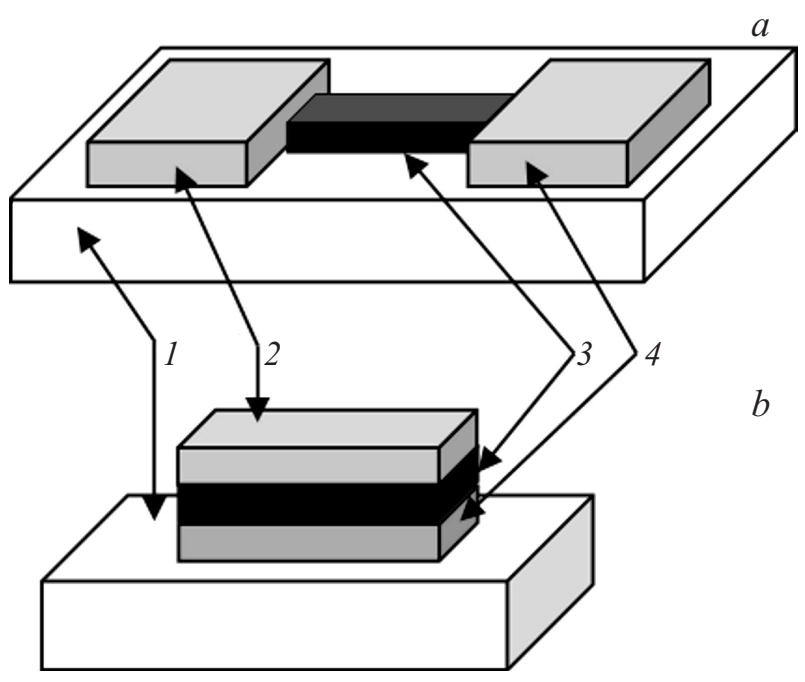

Рис. 1. Однослойная $(a)$ и трехслойная $(b)$ конструкции сенсора: 1 - подложка, 2 - поглотитель, 3 - термоэлектрический слой, 4 - теплоотвод. 
Геометрические размеры и параметры сенсора $\mathrm{W} / \mathrm{CeB}_{6} / \mathrm{W}$

\begin{tabular}{|c|c|c|c|c|c|c|c|c|c|}
\hline $\begin{array}{l}\text { Номер } \\
\text { расчета }\end{array}$ & $\begin{array}{c}X, Y, Z_{1}, \\
\text { мКм }\end{array}$ & $\begin{array}{c}Z_{2}, \\
\text { мКм }\end{array}$ & $\begin{array}{c}Z_{3}, \\
\text { мКм }\end{array}$ & $\begin{array}{l}E, \\
\text { эВ }\end{array}$ & $\begin{array}{c}\Delta T_{m}, \\
10^{-4} \mathrm{~K}\end{array}$ & $\begin{array}{l}t_{m}, \\
\text { пс }\end{array}$ & $\begin{array}{l}\Delta U_{m}, \\
\mathrm{M \kappa B}\end{array}$ & $\begin{array}{l}t_{b}, \\
\text { пс }\end{array}$ & $\begin{array}{c}R, \\
\Gamma \Gamma_{ц}\end{array}$ \\
\hline $1 \mathrm{M}$ & $10,10,1.5$ & 1 & 1 & 1000 & 67.8 & 0.162 & 1.017 & $>4000$ & $<0.2$ \\
\hline $2 \mathrm{M}$ & $10,10,1.5$ & 0.5 & 1 & 1000 & 67.8 & 0.162 & 1.017 & $>2500$ & $<0.4$ \\
\hline $3 \mathrm{M}$ & $10,10,1.5$ & 0.1 & 1 & 1000 & 67.5 & 0.156 & 1.013 & 420 & 2.4 \\
\hline $4 \mathrm{M}$ & $10,10,1.5$ & 0.05 & 1 & 1000 & 67.5 & 0.156 & 1.013 & 186.6 & 5.4 \\
\hline $5 \mathrm{M}$ & $10,10,1.5$ & 0.01 & 1 & 1000 & 56.4 & 0.156 & 0.846 & 4.8 & 208 \\
\hline $6 \mathrm{M}$ & $10,10,1.5$ & 0.01 & 2 & 1000 & 56.4 & 0.156 & 0.846 & 4.8 & 208 \\
\hline $7 \mathrm{M}$ & $10,10,1.5$ & 0.01 & 5 & 1000 & 56.4 & 0.156 & 0.846 & 4.8 & 208 \\
\hline $8 \mathrm{M}$ & $10,10,1.5$ & 0.01 & 1 & 1100 & 62 & 0.156 & 0.93 & 5.1 & 196 \\
\hline $9 \mathrm{M}$ & $10,10,1.5$ & 0.01 & 1 & 900 & 50.7 & 0.156 & 0.761 & 4.5 & 222 \\
\hline $10 \mathrm{M}$ & $10,10,1.5$ & 0.01 & 1 & 1010 & 56.9 & 0.156 & 0.854 & 4.8 & 208 \\
\hline $11 \mathrm{M}$ & $20,20,1.5$ & 0.01 & 1 & 1000 & 56.4 & 0.156 & 0.846 & 3.9 & 256 \\
\hline $12 \mathrm{M}$ & $5,5,1.5$ & 0.01 & 1 & 1000 & 56.4 & 0.156 & 0.846 & 8.7 & 114 \\
\hline $13 \mathrm{M}$ & $10,10,0.5$ & 0.05 & 1 & 100 & 368 & 0.012 & 5.52 & 21.9 & 45.7 \\
\hline $14 \mathrm{M}$ & $10,10,0.5$ & 0.05 & 1 & 110 & 405 & 0.012 & 6.072 & 25.5 & 39.2 \\
\hline $15 \mathrm{M}$ & $10,10,0.5$ & 0.05 & 1 & 90 & 331 & 0.012 & 4.968 & 18 & 55.5 \\
\hline $16 \mathrm{M}$ & $10,10,0.5$ & 0.01 & 1 & 100 & 337 & 0.012 & 5.057 & 3 & 333 \\
\hline $17 \mathrm{M}$ & $10,10,0.5$ & 0.01 & 2 & 100 & 337 & 0.012 & 5.057 & 3 & 333 \\
\hline $18 \mathrm{M}$ & $10,10,0.5$ & 0.01 & 5 & 100 & 337 & 0.012 & 5.057 & 3 & 333 \\
\hline $19 \mathrm{M}$ & $10,10,0.1$ & 0.01 & 1 & 10 & 1147 & 0.0015 & 17.2 & 1.32 & 758 \\
\hline $20 \mathrm{M}$ & $10,10,0.1$ & 0.01 & 1 & 11 & 1261 & 0.0015 & 18.9 & 1.44 & 694 \\
\hline $21 \mathrm{M}$ & $10,10,0.1$ & 0.01 & 1 & 9 & 1033 & 0.0015 & 15.5 & 1.2 & 833 \\
\hline $22 \mathrm{M}$ & $10,10,0.1$ & 0.01 & 1 & 7 & 803 & 0.0015 & 12.05 & 0.963 & 1038 \\
\hline $23 \mathrm{M}$ & $10,10,0.1$ & 0.01 & 1 & 4 & 459 & 0.0015 & 6.885 & 0.651 & 1536 \\
\hline $24 \mathrm{M}$ & $10,10,0.1$ & 0.01 & 1 & 1 & 115 & 0.0015 & 1.725 & 0.303 & 3300 \\
\hline $25 \mathrm{M}$ & $10,10,0.1$ & 0.01 & 1 & 1.1 & 126 & 0.0015 & 1.89 & 0.321 & 3115 \\
\hline $26 \mathrm{M}$ & $10,10,0.1$ & 0.01 & 1 & 0.9 & 103 & 0.0015 & 1.545 & 0.282 & 3546 \\
\hline
\end{tabular}

такого сенсора не требует дополнительного источника мощности или приложенного напряжения, следовательно, дополнительных контактов.

Сенсор TSPD имеет максимально простую конструкцию и обеспечивает возможность создания детекторной матрицы из множества сенсоров с очень простой электронной структурой. Однако такой сенсор имеет недостатки, ограничивающие его применение. Энергия фотона определяется по интегралу временно́й зависимости сигнала. Если время спада сигнала до фонового значения будет больше времени прохождения тепла через границу металл-диэлектрик, то часть выделенного фотоном тепла уйдет в подложку и определить энергию фотона будет невозможно. Энергию фотона нельзя определить и по максимальному значению возникающего сигнала, так как форма временно́й зависимости отклика зависит от области термализации фотона. Исключить эту зависимость и возможность ухода тепла из поглотителя в подложку позволяет трехслойная конструкция сенсора TSPD (рис. 1, $b$ ).

Компьютерное моделирование проводилось на основе уравнения распространения тепла из ограниченного объема с применением трехмерного матричного метода. Подробности использованных подходов и приближений приведены в [5]. Отметим, что в качестве материала поглотителя и теплоотвода нами был выбран воль- фрам (W), а термоэлектриком является гексаборид церия $\left(\mathrm{CeB}_{6}\right)$, имеющий высокий термоэлектрический кпд при $9 \mathrm{~K}$. Именно эта температура определена нами в качестве рабочей для сенсора TSPD.

\section{3. Результаты и обсуждение}

Важнейшим параметром сенсора термоэлектрического детектора является толщина поглотителя. Мы выбирали этот параметр исходя из требования обеспечения большой вероятности поглощения фотона в абсорбере. Воспользовавшись законом Бугера-Ламберта, используя значения коэффициента линейного ослабления в вольфраме для фотонов с энергией 1 кэВ, 100, 10 эВ соответственно $5.775,28.875,115.5$ мкм $^{-1}$ [6], легко рассчитать, что вероятность поглощения фотонов с энергией 1 кэВ в вольфраме толщиной 1.5 мкм будет равна 0.9998. Для фотонов с энергиями 100 и 10 эВ в вольфраме толщиной 0.5 и 0.1 мкм соответственно вероятность поглощения превысит 0.9999.

Геометрические размеры сенсора и результаты расчета процессов распространения тепла в трехслойном сенсоре представлены в таблице. В колонках таблицы приведены: номер расчета, геометрические размеры абсорбера $\left(X, Y, Z_{1}\right)$, толщина термоэлектрического слоя $\left(Z_{2}\right)$, толщина теплоотвода $\left(Z_{3}\right)$, энергия фото- 


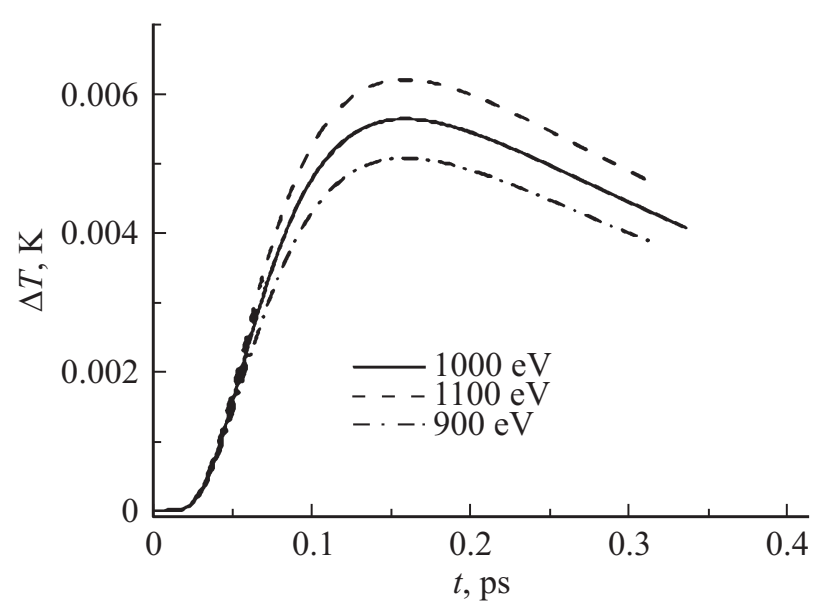

Рис. 2. Зависимости $\Delta T(t)$ по расчетам $5 \mathrm{M}, 8 \mathrm{M}, 9 \mathrm{M}$.

на $(E)$, максимальное значение разности температур, возникающей на термоэлектрике после поглощения фотона $\left(\Delta T_{m}\right)$, время достижения максимальной разности температур $\left(t_{m}\right)$, максимальная величина возникающей на сенсоре разности потенциала $\left(\Delta U_{m}\right)$, время спада сигнала до фонового значения разности темеператур $\Delta T=10^{-4} \mathrm{~K}\left(t_{b}\right)$, обратная ей величина (скорость счета) $R$. В соответствии с рис. $1, b$, геометрические размеры $X$ и $Y$ термоэлектрического слоя и теплоотвода такие же, как у поглотителя. Принималось, что фотон поглощается в центре поверхности поглотителя. Из данных таблицы следует, что для расчета $1 \mathrm{M}$ время спада до уровня фона $>4000$ пс, что определяет малую величину скорости счета. Причиной тому является низкое значение коэффициента теплопроводности $\mathrm{CeB}_{6}$.

Ускорить процесс передачи тепла из поглотителя в теплоотвод можно посредством уменьшения толщины термоэлектрического слоя. В расчетах $1 \mathrm{M}-5 \mathrm{M}$ толщина термоэлектрического слоя последовательно уменьшалась от 1 до 0.01 мкм. От предыдущих существенно отличается расчет 5M, соответствующий толщине термоэлектрика 0.01 мкм. Значения параметров $\Delta T_{m}$ и $\Delta U_{m}$ несколько ниже, однако, как следует из таблицы, сигнал значительно быстрее спадает до фонового значения, обеспечивая скорость счета 200 ГГц. Соответственно эту толщину диэлектрика мы будем использовать в последующих расчетах.

Сравним результаты расчетов 5М-7M, которые проведены для сенсоров с толщиной вольфрамового теплоотвода 1-5мкм. Из таблицы видно, что изменение толщины теплоотвода не оказывает влияния ни на величину максимума сигнала, ни на скорость счета. Можно заключить, что толщина теплоотвода не оказывает значительного влияния на характеристики сенсора и может быть выбрана из других конструкционных требований.

На рис. 2 приведены временны́е зависимости разности температур $\Delta T(t)$ для случаев поглощения фотонов с энергией 1000 и $(1000 \pm 100)$ эВ. Можно видеть, что изменение энергии фотона приводит к изменению значения $\Delta T_{m}$, тогда как величина $t_{m}$ не изменяется. Из данных таблицы следует, что значения $\Delta U_{m}$ различаются на $85 \mathrm{HB}$, если энергии фотонов различаются на $10 \%$, и на $8 \mathrm{HB}$, если энергии фотонов различаются на $1 \%$. Такие значения разности потенциалов можно измерить в эксперименте.

Следующие в таблице расчеты проведены для сенсоров, имеющих по координатам $X$ и $Y$ в 2 раза бо́льшие размеры $(11 \mathrm{M})$ и в 2 раза меньшие размеры (12M). Сравнение их характеристик с характеристиками расчета 5М показывает, что в первом случае скорость счета возрастает от 208 до 256 ГГц, тогда как во втором уменьшается до 114ГГц. При этом значения параметра $\Delta T_{m}$ не различаются. Можно утверждать, что в трехслойном сенсоре TSPD поглотитель выполняет не только задачу превращения энергии фотона в тепловую, но и роль отвода тепла из зоны термализации фотона.

Перейдем к рассмотрению расчетов по детектированию ультрафиолетовых (УФ) фотонов с энергией от 110 эВ, которые граничат с рентгеновскими, до 4 эВ - граница с видимой областью. В расчетах $13 \mathrm{M}-15 \mathrm{M}$ рассматривается поглощение фотонов с энергией 100 и $(100 \pm 10)$ эВ в вольфрамовом поглотителе толщиной 0.5 мкм. Из таблицы следует, что разности потенциалов, соответствующие максимумам этих зависимостей, различаются на 0.55 мкВ. Регистрация сигналов, различающихся на нановольты, позволит говорить о энергетическом разрешении 0.1 эВ.

Представленные в таблице расчеты для фотонов с энергией 11 эВ и меньше проведены для сенсора с толщиной поглотителя 0.1 мкм. На рис. 3 приведена зависимость параметра $\Delta U_{m}$ от энергии поглощенного фотона для расчетов 19M-26М. Наблюдаемая линейность показывает, что параметр $\Delta U_{m}$, т. е. измеряемый в эксперименте максимум возникающей на сенсоре разности потенциалов, может быть использован для точного определения энергии поглощенного фотона.

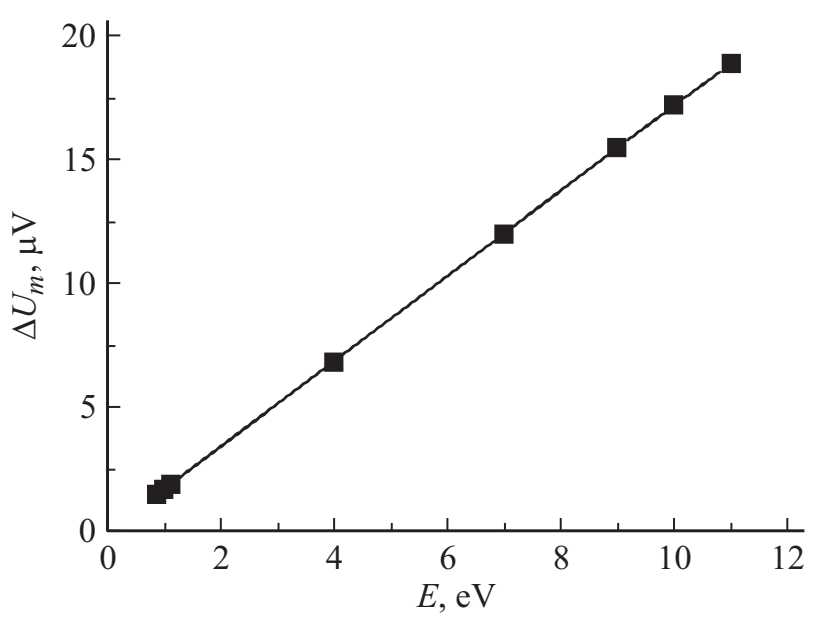

Рис. 3. Зависимость величины параметра $\Delta U_{m}$ от энергии поглощенного фотона (расчеты 19M-26М). 


\section{4. Заключение}

Полученные результаты позволяют сделать следующие выводы. Трехслойный сенсор TSPD может регистрировать одиночные фотоны в широкой области электромагнитного спектра от 1 эВ до 1 кэВ, обеспечить энергетическое разрешение не хуже $1 \%$ и скорость счета от десятков гигагерц до терагерц.

Исследование выполнено при финансовой поддержке ГКН МОН РА (Армения) и РФФИ (Российская Федерация) в рамках совместных научных программ 15RF-018 и 15-53-05047 соответственно.

\section{Список литературы}

[1] R.H. Hadfield. Nature Photonics, 3, 696 (2009).

[2] G.N. Gol'tsman, O. Okunev, G. Chulkova, A. Lipatov, A. Semenov, K. Smirnov, B. Voronov, A. Dzardanov, C. Williams, R. Sobolewski. Appl. Phys. Lett., 79, 705 (2001).

[3] G.G. Fritz, K.S. Wood, D. van Vechten, A.L. Gyulamiryan, A.S. Kuzanyan, N.J. Giordano, T.M. Jacobs, H.-D. Wu, J.S. Horwitz, A.M. Gulian. Proc. SPIE, 4140, 459 (2000).

[4] A. Gulian, K. Wood, D. van Vechten, G. Fritz. J. Mod. Opt., 51, 1467 (2004).

[5] А.А. Кузанян, А.С. Кузанян, В.Р. Никогосян, В.Н. Гурин, М.П. Волков. Изв. НАН Армении. Физика, 51 (2), 244 (2016).

[6] C.T. Chantler. J. Phys. Chem. Ref. Data, 24, 71 (1995).

Редактор Л.В. Шаронова

\section{The perspectives of using rare earths hexaborides in the thermoelectric single-photon detectors}

A.S. Kuzanyan' ${ }^{1}$, A.A. Kuzanyan', V.R. Nikoghosyan', V.N. Gurin' ${ }^{2}$, M.P. Volkov ${ }^{2}$

${ }^{1}$ Institute for Physical Research, National Academy of Sciences of Armenia, 0203 Ashtarak, Armenia

${ }^{2}$ loffe Institute, 194021 St. Petersburg, Russia

Abstract The results of computer simulation of heat distribution processes taking place after absorption of single photons of $1 \mathrm{eV}-1 \mathrm{keV}$ energy in three-layer sensor of the thermoelectric detector are analyzed. Different geometries of the sensor with tungsten absorber, thermoelectric layer of cerium hexaboride and tungsten heat sink are considered. It is shown that by changing the sizes of the sensor layers it is possible to obtain transducers for registration of photons within the given spectral range with required energy resolution and count rate. It is concluded that, as compared to the single layer sensor, the three-layer sensor has a number of advantages and demonstrate characteristics that make possible to consider the thermoelectric detector as a real alternative to superconducting single photon detectors. 